\title{
Towards Deformable Shape Modeling of the Left Atrium Using Non-Rigid Coherent Point Drift Registration
}

\author{
Martin Koch ${ }^{1}$, Sebastian Bauer ${ }^{1}$, Joachim Hornegger ${ }^{1,2}$, Norbert Strobel ${ }^{3}$ \\ ${ }^{1}$ Pattern Recognition Lab, Friedrich-Alexander-Universität Erlangen-Nürnberg \\ ${ }^{2}$ Erlangen Graduate School in Advanced Optical Technologies (SAOT), \\ Friedrich-Alexander-Universität Erlangen-Nürnberg \\ ${ }^{3}$ Siemens AG, Healthcare Sector, Forchheim \\ martin.koch@cs.fau.de
}

\begin{abstract}
Modeling the deformable shape of the left atrium is of strong interest for many applications in cardiac diagnosis and intervention. In this paper, we propose a method for left atrium shape modeling using non-rigid point cloud registration. In particular, we build upon the concept of Coherent Point Drift (CPD) registration that considers the alignment as a probability density estimation problem. Based on the set of non-rigidly registered point clouds, we perform a principle component analysis to establish a deformable shape model. In an experimental study on ten clinical data sets, we evaluated the registration accuracy in terms of average mesh-to-mesh distance, as well as on anatomical landmarks on the left atrium. With the proposed method, we achieved registration results with an average mesh-to-mesh error of $3.4 \mathrm{~mm}$. The average landmark offset was $8.5 \mathrm{~mm}$.
\end{abstract}

\section{Introduction}

Statistical shape models are widely used today in different fields of medical image processing. A common application is the use as prior information for segmentation of 3-D medical image data [1]. The left atrium is a challenging structure, as it shows a large amount of variation in surface topology and shape across different patients. Examples of 3-D mesh models for different atria are shown in Fig. 1. In addition to the anatomical differences among subjects, there is the aspect of cardiac motion which leads to deformations of the left atrium in different heart phases. In electrophysiology ablation procedures, a model of the underlying anatomical structure could be used for planning of the intervention as, e.g., suggested by Keustermans et al. using patient specific 3-D data sets for planning of atrial fibrillation treatment [2].

In this paper, we propose a method for left atrium shape modeling using nonrigid point cloud registration. In our approach, we generate the shape model from 3-D magnetic resonance imaging (MRI) volume data sets. We exclusively used data sets of left atria with four pulmonary veins, which reflects the most common anatomic configuration [3]. First, the relevant structure was segmented and 
represented as a triangle mesh. Then we used the Coherent Point Drift (CPD) algorithm [4] to pairwise align meshes via non-rigid point cloud registration. Basically, CPD registration is performed based on a Gaussian Mixture Model (GMM) framework and a regularization of the displacement field. Benefits of the CPD algorithm are the generation of smooth deformation fields while being robust against noise and outliers [4].

\section{Materials and Methods}

Left atrium mesh models of ten subjects were extracted from contrast enhanced 3 -D MRI volume data sets. The MRI data sets were acquired with a resolution of $256 \times 256 \times 68$ voxels. The in-plane pixel spacing was $1.23 \times 1.23 \mathrm{~mm}$ and the slice thickness $1.5 \mathrm{~mm}$. The left atrium was segmented from MRI voxel data sets using a semi-automatic segmentation software (syngo InSpace EP, Siemens AG, Forchheim, Germany). The segmentation process is initialized by manually selecting a point inside the left atrium. Based on this seedpoint, the complete left atrium is segmented automatically. The segmentation results are represented

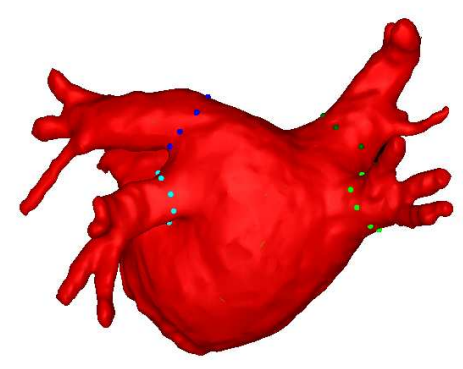

(a)

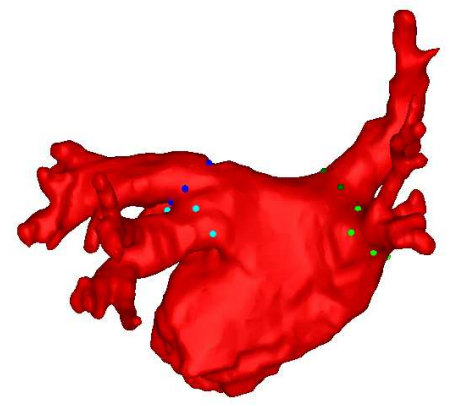

(c)

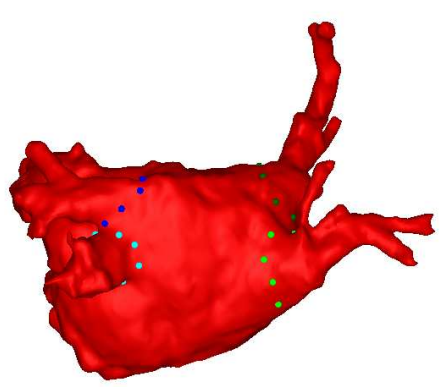

(b)

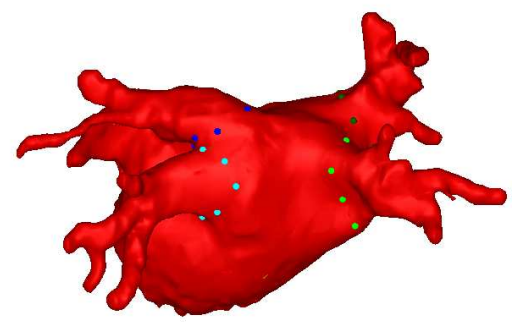

(d)

Fig. 1. Mesh models of the left atrium segmented from 3-D MRI volumes from four subjects (a-d). The colored spheres depict the anatomic position of the pulmonary vein ostia and were labeled by an expert: right superior (dark green), right inferior (light green), left superior (dark blue) and left inferior (light blue). 
as triangle meshes. Fig. 1 depicts models of the left atrium from four different subjects.

For registration, let us consider the mesh as a point cloud $\mathcal{M}$ consisting of $N$ points $\boldsymbol{x}_{i} \in \mathbb{R}^{3}$

$$
\mathcal{M} \equiv \boldsymbol{m}=\left[\boldsymbol{x}_{1}^{\top}, \ldots, \boldsymbol{x}_{N}^{\top}\right]^{\top} \in \mathbb{R}^{3 N}
$$

In a first step, we selected one left atrium mesh model as a reference mesh. The reference mesh was chosen based on visual inspection to clearly express the LA anatomy. The reference mesh $\boldsymbol{m}_{\text {Ref }}$ is then registered to a set of sample meshes $\left\{\boldsymbol{m}_{t}\right\}_{t=1}^{T}$, with $T=9$, using the CPD algorithm. All meshes have the same anatomical orientation, and are zero centered before applying the registration.

\subsection{Non-Rigid Point Cloud Registration Using CPD}

We used the coherent point drift algorithm to register the reference mesh to the set of sample meshes. CPD follows a probabilistic approach by considering the alignment of the two point sets as a probability density estimation problem. The basic idea is to fit the GMM centroids, represented by the points of the reference mesh $\boldsymbol{m}_{\text {Ref }}$, to the sample mesh $\boldsymbol{m}_{t}$, by maximizing the likelihood. This optimization is performed with the expectation maximization algorithm. During the optimization process, the GMM centroids are forced to move coherently as a group, to ensure preservation of the topological structure of the point set.

The displacement function $v$ for the reference mesh is defined as

$$
\hat{\boldsymbol{m}}_{\mathrm{Ref}}=\boldsymbol{m}_{\mathrm{Ref}}+v\left(\boldsymbol{m}_{\mathrm{Ref}}\right)
$$

with $\boldsymbol{m}_{\text {Ref }}$ as the initial centroid positions. $\hat{\boldsymbol{m}}_{\text {Ref }}$ and $v$, respectively, are obtained by minimizing the following energy function [5]:

$$
E\left(\hat{\boldsymbol{m}}_{\mathrm{Ref}}\right)=-\sum_{n=1}^{N} \log \sum_{m=1}^{M} e^{-\frac{1}{2}\left\|\frac{\boldsymbol{x}_{n}-\boldsymbol{y}_{m}}{\sigma}\right\|^{2}}+\frac{\lambda}{2} \phi(v)
$$

where $\phi(v)$ is a regularization to ensure the displacement field to be smooth. $\boldsymbol{x}_{n}$ denotes a point of the mesh $\boldsymbol{m}_{t}, \boldsymbol{y}_{m}$ a point of the transformed mesh $\hat{\boldsymbol{m}}_{\text {Ref }}$, respectively. $N$ and $M$ refer to the number of points within the respective mesh. The parameter $\lambda$ determines the trade-off between data fitting and smoothness of the deformation field. We empirically determined a suitable value for this parameter $(\lambda=2.0)$.

\subsection{Deformable Shape Model Generation}

The reference mesh $\boldsymbol{m}_{\mathrm{Ref}}$ is registered to every sample mesh $\boldsymbol{m}_{t}$. The transformed mesh $\hat{\boldsymbol{m}}_{\mathrm{Ref}}$ is labeled $\boldsymbol{v}_{t}$ for ease of use. The training set is defined as $\mathcal{V}=\left\{\boldsymbol{m}_{\mathrm{Ref}}, \boldsymbol{v}_{1}, \ldots, \boldsymbol{v}_{T}\right\}$. We used a Principle Component Analysis (PCA) approach [6] to compute the modes of variation. Applying PCA to the covariance matrix of the centered version of $\mathcal{V}$ yields a set of eigenvectors $\boldsymbol{e}_{i}$ describing the 
principle modes of variation in the training data set. The eigenvectors are ordered in descending order based on the value of their corresponding eigenvalue. The $P$ largest eigenvectors are stored in the matrix $\boldsymbol{\Phi}=\left[\boldsymbol{e}_{1}, \ldots, \boldsymbol{e}_{P}\right] \in \mathbb{R}^{3 N \times P}$. A linear combination of the $P$ principal modes of variation, with $\boldsymbol{b} \in \mathbb{R}^{P}$ as weighting factors, spans a subset of linearized mesh models composed of the given modes of variation:

$$
\boldsymbol{m}^{*}=\overline{\boldsymbol{v}}+\boldsymbol{\Phi} \boldsymbol{b}
$$

The mean shape $\overline{\boldsymbol{v}}$ is defined as

$$
\overline{\boldsymbol{v}}=\frac{1}{T+1}\left(\boldsymbol{m}_{\mathrm{Ref}}+\sum_{t=1}^{T} \boldsymbol{v}_{t}\right)
$$

For quantitative evaluation of the proposed framework, we used ten clinical data sets with manually annotated pulmonary vein (PV) ostia. These landmarks are labeled RSPV (Right Superior Pulmonary Veins), RIPV (Right Inferior Pulmonary Veins), LSPV (Left Superior Pulmonary Veins), and LIPV (Left Inferior Pulmonary Veins). The quality of the registration is measured based on residual landmark distances and mesh-to-mesh distance. The residual landmark error is defined as the Euclidean distance of the center of corresponding PV ostia and measured after non-rigid CPD registration.

\section{Results}

Quantitative registration results are shown in Fig. 2 and discussed in more detail in Section 4. Fig. 2(a) shows the average mesh-to-mesh distance, as well as the initial and residual landmark offset per data set. The initial and residual offsets per landmark are shown in Fig. 2(b). The average mesh-to-mesh distance is between 2.5 and $5.1 \mathrm{~mm}$, the average landmark offset is between 2.9 and $13.9 \mathrm{~mm}$. Fig. 3 depicts the residual registration error for data set 4, which shows the highest mesh-to-mesh error. The highest mesh-to-mesh distance occurs at the end of the pulmonary veins. In this case especially on the right inferior PV.

\section{Discussion}

We described a method for left atrium shape modeling using non-rigid point cloud registration. The overall performance of the mesh registration shows a mean mesh-to-mesh error of $3.4 \mathrm{~mm}$ over all data sets. The coherent point drift algorithm was capable of dealing with high variations in anatomy. The highest residual mesh-to-mesh distance results from different extents of the pulmonary

veins. The average landmark offset was $8.5 \mathrm{~mm}$. Landmarks on the right side of the left atrium, namely RSPV and RIPV, show a lower residual error compared to left sided landmarks LSPV and LIPV. This might be due to the additional pouch on the left side of the left atrium, the left atrial appendage, which is anterior to the PV ostia. 
The mesh models of the left atrium also contained a large part of the connected pulmonary veins. Removing or trimming these extensions might improve the accuracy, since these structures show a high variation in shape and size. For the modeling of the atrium, short pulmonary vein ostia would be sufficient. This work is a first step towards our goal of automatic planning of ablation regions for atrial fibrillation procedures. Planning structures could be transfered to augmented fluoroscopy systems used to guide the procedure and overlaid to the X-ray images.

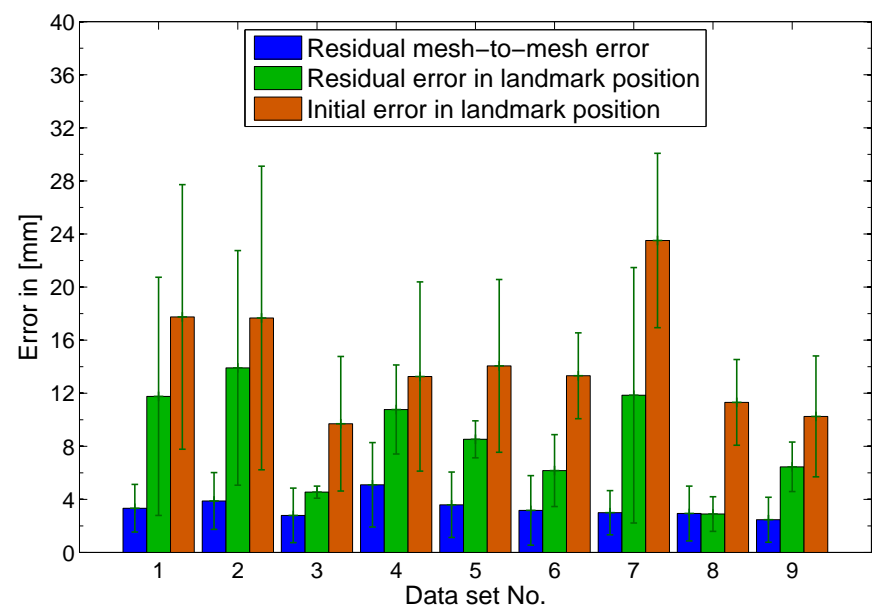

(a) Evaluation per data set.

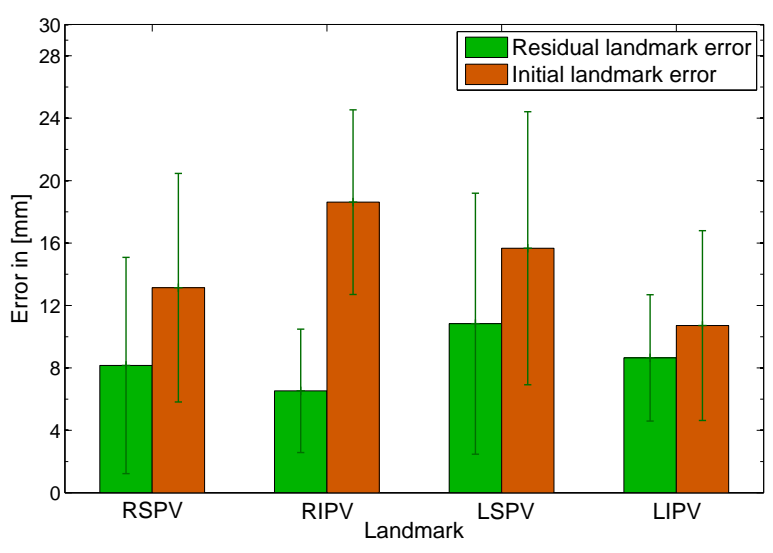

(b) Evaluation per landmark.

Fig. 2. (a) Mean and standard deviation of the residual mesh-to-mesh error, landmark error after registration, and initial landmark error are shown per data set. (b) Mean and standard deviation of the initial and residual landmark error. 
Koch et al.

Fig. 3. Residual mesh-to-mesh error color coded on one example mesh. The error is measured as Euclidean distance in $[\mathrm{mm}]$. The highest mesh-to-mesh distance occurs at the end of the pulmonary veins. In this case especially on the right inferior PV.

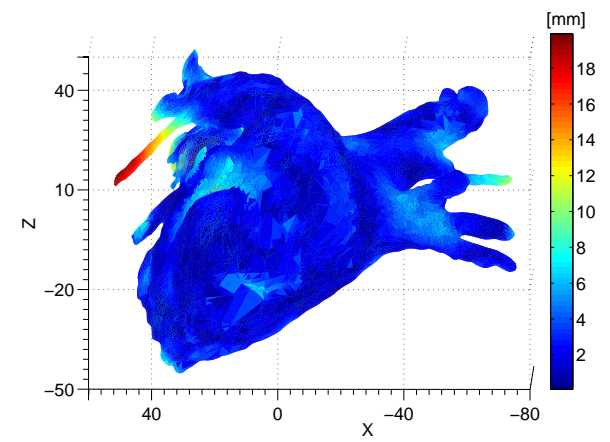

(a) Posterior view

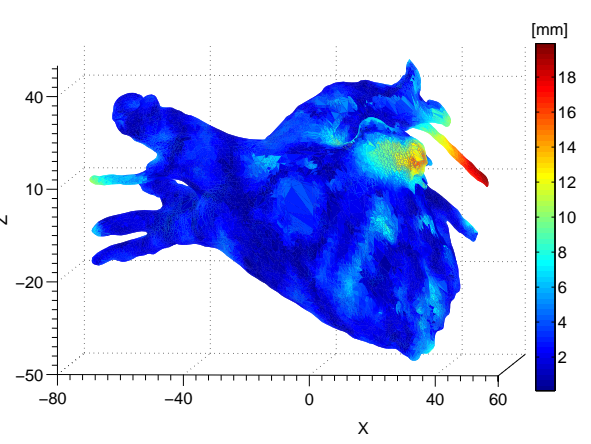

(b) Anterior view

\section{Acknowledgment}

This work was supported by the German Federal Ministry of Education and Research (BMBF) in the context of the initiative Spitzencluster Medical Valley - Europäische Metropolregion Nürnberg, project grant Nos. 01EX1012A and 01EX1012E, respectively. Additional funding was provided by Siemens AG, Healthcare Sector. S. Bauer acknowledges support by the Graduate School of Information Science in Health (GSISH) and TUM Graduate School.

Disclaimer: The concepts and information presented in this paper are based on research and are not commercially available.

\section{References}

1. Heimann T, Meinzer HP. Statistical shape models for 3D medical image segmentation: A review. Med Image Anal. 2009;13(4):543 - 563.

2. Keustermans J, De Buck S, Heidbuechel H, Suetens P. Automated planning of ablation targets in atrial fibrillation treatment. Proc SPIE. 2011;7962:796207.

3. Kautzner J, Micochova H, Peichl P. Anatomy of the Left Atrium and Pulmonary Veins-Lessons Learned from Novel Imaging Techniques. Eur Cardiol. 2006;2(1):8990.

4. Myronenko A, Song X. Point set registration: Coherent Point Drift. IEEE Trans Pattern Anal Mach Intell. 2010;32(12):2262-2275.

5. Myronenko A, Song X, Carreira-Perpinan M. Non-rigid point set registration: Coherent Point Drift. Adv Neural Inf Process Syst. 2007;19:1009-1016.

6. Jolliffe I. Principal component analysis. Wiley Online Library; 2005. 